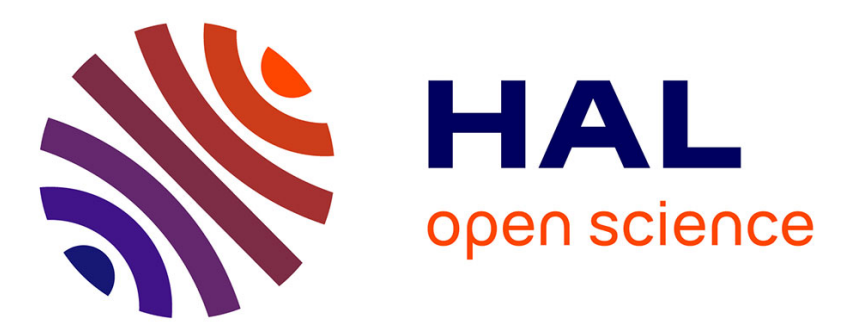

\title{
PRINCIPAL EIGENVALUES FOR SYSTEMS OF SCHRÖDINGER EQUATIONS DEFINED IN THE WHOLE SPACE WITH INDEFINITE WEIGHTS
}

Laure Cardoulis

\section{- To cite this version:}

Laure Cardoulis. PRINCIPAL EIGENVALUES FOR SYSTEMS OF SCHRÖDINGER EQUATIONS DEFINED IN THE WHOLE SPACE WITH INDEFINITE WEIGHTS. Mathematica Slovaca, 2015. hal-03436163

\section{HAL Id: hal-03436163 \\ https://hal.science/hal-03436163}

Submitted on 19 Nov 2021

HAL is a multi-disciplinary open access archive for the deposit and dissemination of scientific research documents, whether they are published or not. The documents may come from teaching and research institutions in France or abroad, or from public or private research centers.
L'archive ouverte pluridisciplinaire HAL, est destinée au dépôt et à la diffusion de documents scientifiques de niveau recherche, publiés ou non, émanant des établissements d'enseignement et de recherche français ou étrangers, des laboratoires publics ou privés. 


\title{
PRINCIPAL EIGENVALUES FOR SYSTEMS OF SCHRÖDINGER EQUATIONS DEFINED IN THE WHOLE SPACE WITH INDEFINITE WEIGHTS
}

\author{
LAURE CARDOULIS*
}

\begin{abstract}
We present in this paper some results for the existence of principal eigenvalues for equations or systems defined in $\mathbb{R}^{N}$ involving Schrödinger operators with indefinite weight functions and with potentials which tend to infinity at infinity.
\end{abstract}

\section{INTRODUCTION}

We consider the eigenvalue problem for the following system:

$$
\left(-\Delta+q_{i}\right) u_{i}=\lambda\left(m_{i} u_{i}+\sum_{j=1 ; j \neq i}^{n} m_{i j} u_{j}\right) \text { in } \mathbb{R}^{N}, i=1, \cdots, n .
$$

We consider the following hypothesis for each $i=1, \ldots, n$ :

$\left(\mathbf{H}_{\mathbf{q}}^{\mathbf{1}}\right): q_{i} \in L_{l o c}^{2}\left(\mathbb{R}^{N}\right) \cap L_{l o c}^{p}\left(\mathbb{R}^{N}\right), p>\frac{N}{2}$, such that $\lim _{|x| \rightarrow \infty} q_{i}(x)=\infty$ and $q_{i} \geq c s t>0$.

We will later specify the hypotheses on each weight $m_{i}$ and on each function $m_{i j}$ and we denote by $\lambda$ a real parameter. The variational space is denoted by

$$
V:=V_{q_{1}}\left(\mathbb{R}^{N}\right) \times \cdots \times V_{q_{n}}\left(\mathbb{R}^{N}\right),
$$

where for each $i=1, \ldots, n, V_{q_{i}}\left(\mathbb{R}^{N}\right)$ is the completion of $D\left(\mathbb{R}^{N}\right)$, the set of $\mathcal{C}^{\infty}$ functions with compact supports, with respect to the norm

$$
\|u\|_{q_{i}}^{2}=\int_{\mathbb{R}^{N}}\left[|\nabla u|^{2}+q_{i} u^{2}\right] .
$$

We recall that the embedding of each $V_{q_{i}}\left(\mathbb{R}^{N}\right)$ into $L^{2}\left(\mathbb{R}^{N}\right)$ is compact.

The aim of this paper is mainly to study the existence of principal eigenvalues for the system (1.1) with indefinite weights $m_{i}$ which are not necessarily bounded and with coefficients $m_{i j}$ which are also not necessarily bounded. This extends earlier results obtained either for the Laplacian operator in a bounded domain (see [5], [15], [17]), or for the Laplacian operator with an indefinite weight in $\mathbb{R}^{N}$ (see [4]), or for equations involving Schrödinger operators $-\Delta+q_{i}$ in $\mathbb{R}^{N}$ with indefinite and non necessarily bounded weights (see [6]), or for systems involving Schrödinger operators $-\Delta+q_{i}$ in $\mathbb{R}^{N}$ with indefinite and bounded weights and with bounded coefficients (see [7]).

1991 Mathematics Subject Classification. (AMS) 35J10.

Key words and phrases. Schrödinger operator, indefinite weight, principal eigenvalue. 
We rewrite the system (1.1) under the following form

$$
L U=\lambda M U
$$

where $L$ is the diagonal matrix given by $L=\operatorname{diag}\left(-\Delta+q_{i}\right),{ }^{t} U=u=\left(u_{1}, \cdots, u_{n}\right)$ and $M$ is the $n \times n$-matrix given by $M=\left(m_{i j}\right)$ with $m_{i i}:=m_{i}$.

Our main result concerns the existence and uniqueness of a positive, global and principal eigenvalue $\Lambda_{1, M}$ for (1.2) with a cooperative and symmetric matrix $M$ (i.e. $m_{i j}>0$ and $m_{i j}=m_{j i}$ for all $i \neq j$ ) and with indefinite weight $m_{i} \in$ $L^{N / 2}\left(\mathbb{R}^{N}\right) \cap L^{N}\left(\mathbb{R}^{N}\right) \cap L_{\text {loc }}^{\infty}\left(\mathbb{R}^{N}\right)(N \geq 3)$ and non necessarily bounded coefficients $m_{i j}$. We also obtain a Courant-Fischer formula for this positive principal eigenvalue $\Lambda_{1, M}$.

Our paper is organized as follows: In Section 2 we recall some results for the scalar case, i.e. for the eigenvalue problem $(-\Delta+q) u=\lambda m u$ in $\mathbb{R}^{N}$ where $q$ is a potential which satisfies $\left(\mathbf{H}_{\mathbf{q}}^{\mathbf{1}}\right)$ and where $m$ is an indefinite weight, non necessarily bounded. We also recall some results for the cooperative system (1.2) in the case of indefinite and bounded weights (see [7, Theorems 2.5,2.6]). The Section 3 is devoted to the study of the eigenvalue problem for the cooperative system (1.2) in the case of indefinite weights $m_{i} \in L^{N / 2}\left(\mathbb{R}^{N}\right) \cap L^{N}\left(\mathbb{R}^{N}\right) \cap L_{l o c}^{\infty}\left(\mathbb{R}^{N}\right)(N \geq 3)$. First for positive weights $m_{i}$, as in $[6,7,15]$, we obtain the existence and uniqueness of a positive principal eigenvalue $\Lambda_{1, M}$ by using a general version of the Krein-Rutman Theorem given in [11] which allows us to work in $L^{2^{*}}\left(\mathbb{R}^{N}\right)$, where $2^{*}=\frac{2 N}{N-2}$, whose positive cone has empty interior. We also obtain a Courant-Fischer formula for $\Lambda_{1, M}$. Then for indefinite weights $m_{i}$, we adapt the ideas expressed in $[15,6,7]$ to our case by rewriting (1.2) in an equivalent form with positive weights and a parameter function $\sigma$ depending upon $\lambda$. Then using the properties of the function $\sigma$ and the variational characterization of the corresponding principal eigenvalue obtained in the case of positive weights, we can get the existence and uniqueness of a positive and principal eigenvalue for (1.2). Moreover we can compare $\Lambda_{1, M}$ to each principal eigenvalue of $-\Delta+q_{i}$ associated with $m_{i}$ in $\mathbb{R}^{N}$. So in Section 3 we extend some results already obtained for bounded weights and bounded coefficients to non necessarily bounded weights and non necessarily bounded coefficients. We conclude this paper by the Section 4 where we give two examples of studies of the existence of a principal eigenvalue for a two-by-two system which is non cooperative.

Finally note that, as in [7], we can get the classical maximum principal result for (1.1) and the existence of solutions for linear systems (see for example the maximum principle and its extension known as the fundamental positivity in $[1,2,3,6,7,12$, $14,18,19]$ and also the antimaximum principle and the fundamental negativity in $[2,9,7,16,19])$.

\section{REVIEW OF RESULTS}

2.1. Review of results for the scalar case. We recall in this section some results for the eigenvalue problem for the Schrödinger operator $-\Delta+q$ associated with the weight $m$. We assume that $q$ is a potential which satisfies $\left(\mathbf{H}_{\mathbf{q}}^{\mathbf{1}}\right)$. We will add another hypothesis upon the potential $q$ which assures that any element of the weak domain 
of the operator $-\Delta+q$ belongs to the strong domain $D(-\Delta+q)$. It is the following hypothesis.

$\left(\mathbf{H}_{\mathbf{q}}^{\mathbf{2}}\right)$ : Any element of the weak domain of the operator $-\Delta+q$ belongs to the strong domain $D(-\Delta+q)$.

$\left(\mathbf{H}_{\mathbf{q}}^{\mathbf{3}}\right):$ For all $x \in \mathbb{R}^{N}$ and all $h \in \mathbb{R}^{N}, h \neq 0,\left|\frac{q(x+h)-q(x)}{h}\right| \leq \operatorname{cst} \sqrt{q(x)}$.

Note that for example, the potential $q(x)=1+|x|$ satisfies $\left(\mathbf{H}_{\mathbf{q}}^{\mathbf{3}}\right)$. Then we have the following lemma based on the methods of translations due to Nirenberg.

Lemma 2.1. ([7, Lemma 2.1]) Assume that the potential $q$ satisfies $\left(\mathbf{H}_{\mathbf{q}}^{\mathbf{1}}\right)$ and $\left(\mathbf{H}_{\mathbf{q}}^{\mathbf{3}}\right)$. Let $u$ be a weak solution of $(-\Delta+q) u=f$ in $\mathbb{R}^{N}$ with $f \in L^{2}\left(\mathbb{R}^{N}\right)$. Then $u \in H^{2}\left(\mathbb{R}^{N}\right), q u \in L^{2}\left(\mathbb{R}^{N}\right)$ and therefore $u \in D(-\Delta+q)$. So q satisfies $\left(\mathbf{H}_{\mathbf{q}}^{\mathbf{2}}\right)$.

The weight $m$ will assume one of the following hypotheses:

$\left(\mathbf{H}_{\mathbf{m}}^{* \mathbf{1}}\right): 0<m \leq$ cst in $\mathbb{R}^{N}$.

$\left(\mathbf{H}_{\mathbf{m}}^{\mathbf{2}}\right): m \in L^{N / 2}\left(\mathbb{R}^{N}\right) \cap L_{\text {loc }}^{\infty}\left(\mathbb{R}^{N}\right)(N \geq 3), m \geq 0, m \neq 0$.

$\left(\mathbf{H}_{\mathbf{m}}^{\mathbf{1}}\right): m \in L^{\infty}\left(\mathbb{R}^{N}\right), m$ is positive in the open subset $\Omega_{m}^{+}=\left\{x \in \mathbb{R}^{N}, m(x)>\right.$ $0\}$ with non zero measure and $m$ is negative in the open subset $\Omega_{m}^{-}=\{x \in$ $\left.\mathbb{R}^{N}, m(x)<0\right\}$ with non zero measure.

$\left(\mathbf{H}_{\mathbf{m}}^{\mathbf{2}}\right): m \in L^{N / 2}\left(\mathbb{R}^{N}\right) \cap L_{\text {loc }}^{\infty}\left(\mathbb{R}^{N}\right)(N \geq 3), m$ is positive in the open subset $\Omega_{m}^{+}$with non zero measure and $m$ is negative in the open subset $\Omega_{m}^{-}$with non zero measure.

For a positive weight we have:

Theorem 2.1. (cf $\left[6\right.$, Theorems 2.1,2.2]) Assume that $q$ satisfies $\left(\mathbf{H}_{\mathbf{q}}^{\mathbf{1}}\right)$ and $m$ satisfies $\left(\mathbf{H}_{\mathbf{m}}^{* \mathbf{1}}\right)$ or $\left(\mathbf{H}_{\mathbf{m}}^{\mathbf{2}}\right)$. Then there exists a unique principal eigenvalue $\lambda_{1, q, m}$ which is simple and associated with a positive eigenfunction $\phi_{1, q, m}$ and:

$$
\begin{gathered}
(-\Delta+q) \phi_{1, q, m}=\lambda_{1, q, m} m \phi_{1, q, m} \text { in } \mathbb{R}^{N} ; \quad \lambda_{1, q, m}>0 ; \quad \phi_{1, q, m}>0 . \\
\lambda_{1, q, m}=\inf \left\{\frac{\int_{\mathbb{R}^{N}}\left[|\nabla \phi|^{2}+q \phi^{2}\right]}{\int_{\mathbb{R}^{N}} m \phi^{2}}, \phi \in V_{q}\left(\mathbb{R}^{N}\right) \text { s.t. } \int_{\mathbb{R}^{N}} m \phi^{2}>0\right\} .
\end{gathered}
$$

For a weight $m$ which changes sign in $\mathbb{R}^{N}$, we have:

Theorem 2.2. (cf $\left[6\right.$, Theorem 3.1]) Assume that $q$ satisfies $\left(\mathbf{H}_{\mathbf{q}}^{\mathbf{1}}\right),\left(\mathbf{H}_{\mathbf{q}}^{\mathbf{3}}\right)$ and $m$ satisfies $\left(\mathbf{H}_{\mathbf{m}}^{\mathbf{1}}\right)$ or $\left(\mathbf{H}_{\mathbf{m}}^{\mathbf{2}}\right)$. Then the operator $-\Delta+q$ associated with the weight $m$ has a unique positive principal eigenvalue $\lambda_{1, q, m}$ associated with a positive eigenfunction $\phi_{1, q, m}$ and $\left(\lambda_{1, q, m}, \phi_{1, q, m}\right)$ satisfy (2.1) and (2.2). Moreover the operator $-\Delta+$ $q$ associated with the weight $m$ has a unique negative principal eigenvalue $\tilde{\lambda}_{1, q, m}$ associated with a positive eigenfunction $\tilde{\phi}_{1, q, m}$ and $\left(\tilde{\lambda}_{1, q, m}, \tilde{\phi}_{1, q, m}\right)$ satisfy

$$
\begin{gathered}
(-\Delta+q) \tilde{\phi}_{1, q, m}=\tilde{\lambda}_{1, q, m} m \tilde{\phi}_{1, q, m} \text { in } \mathbb{R}^{N} ; \quad \tilde{\lambda}_{1, q, m}<0 ; \quad \tilde{\phi}_{1, q, m}>0 . \\
\tilde{\lambda}_{1, q, m}=\sup \left\{\frac{\int_{\mathbb{R}^{N}}\left[|\nabla \phi|^{2}+q \phi^{2}\right]}{\int_{\mathbb{R}^{N}} m \phi^{2}}, \phi \in V_{q}\left(\mathbb{R}^{N}\right) \text { s. t. } \int_{\mathbb{R}^{N}} m \phi^{2}<0\right\} .
\end{gathered}
$$


2.2. Review of results for systems. We recall in this section some results for the existence of a positive principal eigenvalue when the cooperative system (1.1) has bounded weights. So we consider the eigenvalue problem for the system

$$
\left(-\Delta+q_{i}\right) u_{i}=\lambda\left(m_{i} u_{i}+\sum_{j=1 ; j \neq i}^{n} m_{i j} u_{j}\right) \text { in } \mathbb{R}^{N}, i=1, \cdots, n,
$$

where each of the potentials $q_{i}$ satisfy $\left(\mathbf{H}_{\mathbf{q}}^{\mathbf{1}}\right)$ and each of the weights $m_{i}$ satisfy one of the hypotheses among $\left(\mathbf{H}_{\mathbf{m}}^{* \mathbf{1}}\right)$ and $\left(\mathbf{H}_{\mathbf{m}}^{\mathbf{1}}\right)$. We denote by $M$ the $n \times n$-matrix given by $M=\left(m_{i j}\right)$ with $m_{i i}:=m_{i}$. We will consider the following hypotheses:

(H3): For all $i \neq j, m_{i j} \in L^{\infty}\left(\mathbb{R}^{N}\right)$ and $m_{i j}>0$.

(H4): $M$ is a symmetric matrix.

(H5): $\Omega:=\cap_{i=1}^{n} \Omega_{i}^{+}$is an open subset of $\mathbb{R}^{N}$ with non zero measure and with $\Omega_{i}^{+}:=\left\{x \in \mathbb{R}^{N}, m_{i}(x)>0\right\}$.

For positive bounded weights, we have:

Theorem 2.3. [7, Theorem 2.5] Assume that each of the potentials $q_{i}$ satisfy $\left(\mathbf{H}_{\mathbf{q}}^{\mathbf{1}}\right)$ and each of the weights $m_{i}$ satisfy $\left(\mathbf{H}_{\mathbf{m}}^{* \mathbf{1}}\right)$. Assume also that $(\mathbf{H} \mathbf{3})$ is satisfied. Then there exists a unique principal eigenvalue $\Lambda_{1, M}>0$ associated with a positive eigenfunction $\Phi_{1, M}=\left(\phi_{1, M}, \cdots, \phi_{n, M}\right) \in V:=V_{q_{1}}\left(\mathbb{R}^{N}\right) \times \cdots \times V_{q_{n}}\left(\mathbb{R}^{N}\right)$ for the system (1.1). Moreover if $(\mathbf{H 4})$ and $\left(\mathbf{H}_{\mathbf{q}}^{\mathbf{2}}\right)$ are satisfied then

$$
\begin{gathered}
\Lambda_{1, M}=\inf \left\{\frac{\sum_{i=1}^{n}\left\|u_{i}\right\|_{q_{i}}^{2}}{\sum_{i=1}^{n} \int_{\mathbb{R}^{N}} m_{i} u_{i}^{2}+\sum_{i, j ; i \neq j}^{n} \int_{\mathbb{R}^{N}} m_{i j} u_{i} u_{j}}, u=\left(u_{1}, \cdots, u_{n}\right) \in V\right. \\
\text { such that } \left.\sum_{i=1}^{n} \int_{\mathbb{R}^{N}} m_{i} u_{i}^{2}+\sum_{i, j ; i \neq j}^{n} \int_{\mathbb{R}^{N}} m_{i j} u_{i} u_{j}>0\right\} .
\end{gathered}
$$

Note that the condition $\sum_{i=1}^{n} \int_{\mathbb{R}^{N}} m_{i} u_{i}^{2}+\sum_{i, j ; i \neq j}^{n} \int_{\mathbb{R}^{N}} m_{i j} u_{i} u_{j}>0$ is automatically satisfied if $M$ is a definite positive matrix (i. e. for all $X \neq 0,{ }^{t} X M X>0$ ).

For indefinite bounded weights, we have:

Theorem 2.4. [7, Theorem 2.6] Assume that each of the potentials $q_{i}$ satisfy $\left(\mathbf{H}_{\mathbf{q}}^{\mathbf{1}}\right)$ and $\left(\mathbf{H}_{\mathbf{q}}^{\mathbf{2}}\right)$ and each of the weights $m_{i}$ satisfy $\left(\mathbf{H}_{\mathbf{m}}^{\prime \mathbf{1}}\right)$. Assume also that $(\mathbf{H 3})-(\mathbf{H 5})$ are satisfied. Then there exists a unique principal eigenvalue $\Lambda_{1, M}>0$ associated with a positive eigenfunction $\Phi_{1, M}=\left(\phi_{1, M}, \cdots, \phi_{n, M}\right) \in V:=V_{q_{1}}\left(\mathbb{R}^{N}\right) \times \cdots \times$ $V_{q_{n}}\left(\mathbb{R}^{N}\right), \phi_{i, M}>0$ and $\Lambda_{1, M}$ satisfies $(2.3)$.

\section{Existence of a global positive eigenvalue}

In this section $(N \geq 3)$, we consider the eigenvalue problem for the system (1.1)

$$
\left(-\Delta+q_{i}\right) u_{i}=\lambda\left(m_{i} u_{i}+\sum_{j=1 ; j \neq i}^{n} m_{i j} u_{j}\right) \text { in } \mathbb{R}^{N}, i=1, \cdots, n,
$$

where each of the potentials $q_{i}$ satisfy $\left(\mathbf{H}_{\mathbf{q}}^{\mathbf{1}}\right)$ and $\left(\mathbf{H}_{\mathbf{q}}^{\mathbf{2}}\right)$ and each of the weights $m_{i}$ satisfy $\left(\mathbf{H}_{\mathbf{m}}^{\mathbf{3}}\right)$ or $\left(\mathbf{H}_{\mathbf{m}}^{\mathbf{3}}\right)$. We will consider the following hypotheses:

$\left(\mathbf{H}_{\mathbf{m}}^{\mathbf{3}}\right): m \in L^{N}\left(\mathbb{R}^{N}\right) \cap\left(L^{N / 2}\left(\mathbb{R}^{N}\right) \cup L^{\infty}\left(\mathbb{R}^{N}\right)\right) \cap L_{\text {loc }}^{\infty}\left(\mathbb{R}^{N}\right)(N \geq 3), m>0$. 
$\left(\mathbf{H}_{\mathbf{m}}^{\mathbf{3}}\right): m \in L^{N}\left(\mathbb{R}^{N}\right) \cap\left(L^{N / 2}\left(\mathbb{R}^{N}\right) \cup L^{\infty}\left(\mathbb{R}^{N}\right)\right) \cap L_{\text {loc }}^{\infty}\left(\mathbb{R}^{N}\right)(N \geq 3), m$ is positive in the open subset $\Omega_{m}^{+}$with non zero measure and $m$ is negative in the open subset $\Omega_{m}^{-}$with non zero measure.

(H6): For all $i \neq j, m_{i j} \in L^{N}\left(\mathbb{R}^{N}\right) \cap\left(L^{N / 2}\left(\mathbb{R}^{N}\right) \cup L^{\infty}\left(\mathbb{R}^{N}\right)\right) \cap L_{\text {loc }}^{\infty}\left(\mathbb{R}^{N}\right)$ and $m_{i j}>0$.

For strictly positive weights, we can prove the existence of a positive eigenvalue associated with a positive eigenfunction for (1.1).

Theorem 3.1. Assume that each of the potentials $q_{i}$ satisfy $\left(\mathbf{H}_{\mathbf{q}}^{\mathbf{1}}\right)$ and each of the weights $m_{i}$ satisfy $\left(\mathbf{H}_{\mathbf{m}}^{\mathbf{3}}\right)$. Assume also that $(\mathbf{H 6})$ is satisfied. Then there exists a unique principal eigenvalue $\Lambda_{1, M}>0$ associated with a positive eigenfunction $\Phi_{1, M}=\left(\phi_{1, M}, \cdots, \phi_{n, M}\right) \in V:=V_{q_{1}}\left(\mathbb{R}^{N}\right) \times \cdots \times V_{q_{n}}\left(\mathbb{R}^{N}\right)$ for the system $(1.1)$. Moreover if $(\mathbf{H 4})$ and $\left(\mathbf{H}_{\mathbf{q}}^{\mathbf{2}}\right)$ are satisfied then $\Lambda_{1, M}$ satisfies (2.3).

Proof. We denote by $M:\left(\left(L^{2^{*}}\left(\mathbb{R}^{N}\right)\right)^{n},\|\cdot\|_{\left(L^{2^{*}}\left(\mathbb{R}^{N}\right)\right)^{n}}\right) \rightarrow\left(\left(L^{2}\left(\mathbb{R}^{N}\right)\right)^{n},\|\cdot\|_{\left.\left(L^{2}\left(\mathbb{R}^{N}\right)\right)^{n}\right)}\right.$ the operator of multiplication by the matrix $M$. The operator $M$ is well-defined since the coefficients of the matrix are in $L^{N}\left(\mathbb{R}^{N}\right)$. Let the operator

$$
L^{-1} M:\left(\left(L^{2^{*}}\left(\mathbb{R}^{N}\right)\right)^{n},\|\cdot\|_{\left(L^{2^{*}}\left(\mathbb{R}^{N}\right)\right)^{n}}\right) \rightarrow\left(\left(L^{2}\left(\mathbb{R}^{N}\right)\right)^{n},\|\cdot\|_{\left(L^{2}\left(\mathbb{R}^{N}\right)\right)^{n}}\right) .
$$

The operator $L^{-1} M$ is compact because of the compact embedding of $V$ into $\left(L^{2}\left(\mathbb{R}^{N}\right)\right)^{n}$ and, due to the strong maximum principle for each operator $-\Delta+q_{i}$ in $\mathbb{R}^{N}$ and the hypotheses $\left(\mathbf{H}_{\mathbf{m}}^{\mathbf{3}}\right)$ and $(\mathbf{H 6})$, is also strongly positive in the sense of quasi-interior points in $\left(L^{2^{*}}\left(\mathbb{R}^{N}\right)\right)^{n}$, in the sense of Daners and Koch-Medina. This implies that $L^{-1} M$ is irreducible and we apply the version of the Krein-Rutman Theorem given in [11, Theorem 12.3] to deduce that $r\left(L^{-1} M\right)$, the spectral radius of $L^{-1} M$, is a strictly positive and simple eigenvalue associated with an eigenfunction $\Phi_{1, M}=\left(\phi_{1, M}, \cdots, \phi_{n, M}\right)$ which is a quasi-interior point of $\left(L^{2^{*}}\left(\mathbb{R}^{N}\right)\right)^{n}$, that is $\phi_{i, M}>0$ in $\mathbb{R}^{N}$ for all $i$. Of course $\Lambda_{1, M}=\frac{1}{r\left(L^{-1} M\right)}>0$ and $r\left(L^{-1} M\right)$ is the only one eigenvalue of $L^{-1} M$ associated with a positive eigenfunction.

We recall that $V:=V_{q_{1}}\left(\mathbb{R}^{N}\right) \times \cdots \times V_{q_{n}}\left(\mathbb{R}^{N}\right)$ and the inner product in $V$ is defined by $<u, v>_{V}=\sum_{i=1}^{n}<u_{i}, v_{i}>_{q_{i}}$ for all $u=\left(u_{1}, \cdots, u_{n}\right) \in V$ and $v=\left(v_{1}, \cdots, v_{n}\right) \in$ $V$. We set the bilinear form

$$
\beta(u, v)=\sum_{i=1}^{n} \int_{\mathbb{R}^{N}} m_{i} u_{i} v_{i}+\sum_{i, j=1 ; i \neq j}^{n} \int_{\mathbb{R}^{N}} m_{i j} u_{j} v_{i} \text { for all } u \in V \text { and } v \in V .
$$

Note that $H^{1}\left(\mathbb{R}^{N}\right) \subset L^{2^{*}}\left(\mathbb{R}^{N}\right)(N \geq 3)$ with a continuous embedding. Therefore from hypotheses $(\mathbf{H 4})$ and $(\mathbf{H 6}), \beta$ is a bilinear, symmetric and continous form. From the Riesz Theorem, we get the existence of a continuous linear operator $T: V \rightarrow V, T=\left(T_{1}, \cdots, T_{n}\right)$, such that $\beta(u, v)=<T u, v>_{V}$ for all $u \in V$ and $v \in V$.

Now we prove that the operator $T$ is compact.

Let $\left(u_{p}\right)_{p}=\left(u_{1 p}, \cdots, u_{n p}\right)$ be a bounded sequence in $V$. We want to show that there exists a convergent subsequence of $\left(T_{i} u_{p}\right)_{p}$ which is a Cauchy sequence for 
$\|\cdot\|_{q_{i}}$. We have:

$\left\|T_{i} u_{p}-T_{i} u_{l}\right\|_{q_{i}}^{2}=\int_{\mathbb{R}^{N}} m_{i}\left(u_{i p}-u_{i l}\right)\left(T_{i} u_{p}-T_{i} u_{l}\right)+\sum_{j=1 ; j \neq i}^{n} \int_{\mathbb{R}^{N}} m_{i j}\left(u_{j p}-u_{j l}\right)\left(T_{i} u_{p}-T_{i} u_{l}\right)$.

If the weight $m_{i} \in L^{N / 2}\left(\mathbb{R}^{N}\right)$ (similarly the coefficient $m_{i j}$ ) then:

$\int_{\mathbb{R}^{N}} m_{i}\left(u_{i p}-u_{i l}\right)\left(T_{i} u_{p}-T_{i} u_{l}\right) \leq\left(\int_{\mathbb{R}^{N}}\left|m_{i}\left(u_{i p}-u_{i l}\right)\right|^{\frac{2 N}{N+2}}\right)^{\frac{N+2}{2 N}}\left(\int_{\mathbb{R}^{N}}\left|T_{i} u_{p}-T_{i} u_{l}\right|^{\frac{2 N}{N-2}}\right)^{\frac{N-2}{2 N}}$.

Since $N \geq 3$, the embedding of $H^{1}\left(\mathbb{R}^{N}\right)$ into $L^{2^{*}}\left(\mathbb{R}^{N}\right)$ is continuous where $2^{*}=\frac{2 N}{N-2}$ and so the embedding of $V_{q_{i}}\left(\mathbb{R}^{N}\right)$ into $L^{2^{*}}\left(\mathbb{R}^{N}\right)$ is continuous too. Therefore we get the existence of a positive constant $k$ such that:

$$
\int_{\mathbb{R}^{N}} m_{i}\left(u_{i p}-u_{i l}\right)\left(T_{i} u_{p}-T_{i} u_{l}\right) \leq k\left\|m_{i}\left(u_{i p}-u_{i l}\right)\right\|_{L^{\frac{2 N}{N+2}}\left(\mathbb{R}^{N}\right)}\left\|T_{i} u_{p}-T_{i} u_{l}\right\|_{q_{i}} .
$$

Moreover, since the sequence $\left(u_{i p}\right)_{p}$ is bounded in $V_{q_{i}}\left(\mathbb{R}^{N}\right)$, we deduce that the sequence $\left(u_{i p}\right)_{p}$ is bounded in $H^{1}\left(B_{R}\right)$ for all ball $B_{R}=\left\{x \in \mathbb{R}^{N},\|x\|<R\right\}$. Since $B_{R}$ is bounded, the embedding of $H^{1}\left(B_{R}\right)$ into $L^{2}\left(B_{R}\right)$ is compact and we get that $\left(u_{i p}\right)_{p}$ admits a convergent subsequence in $L^{2}\left(B_{R}\right)$ and therefore in $L^{\frac{2 N}{N+2}}\left(B_{R}\right)$ (because of the continuous embedding of $L^{2}\left(B_{R}\right)$ into $L^{\frac{2 N}{N+2}}\left(B_{R}\right)$ ). Let $\epsilon>0$. We have:

$\left\|m_{i}\left(u_{i p}-u_{i l}\right)\right\|_{L^{\frac{2 N}{N+2}}\left(\mathbb{R}^{N}\right)}=\left(\int_{B_{R}}\left|m_{i}\left(u_{i p}-u_{i l}\right)\right|^{\frac{2 N}{N+2}}+\int_{\mathbb{R}^{N}-B_{R}}\left|m_{i}\left(u_{i p}-u_{i l}\right)\right|^{\frac{2 N}{N+2}}\right)^{\frac{N+2}{2 N}}$.

Since $(x+y)^{1 / p} \leq x^{1 / p}+y^{1 / p}$ for all $p>1, x, y>0$ we get:

$\left\|m_{i}\left(u_{i p}-u_{i l}\right)\right\|_{L^{\frac{2 N}{N+2}\left(\mathbb{R}^{N}\right)}} \leq\left(\int_{B_{R}}\left|m_{i}\left(u_{i p}-u_{i l}\right)\right|^{\frac{2 N}{N+2}}\right)^{\frac{N+2}{2 N}}+\left(\int_{\mathbb{R}^{N}-B_{R}}\left|m_{i}\left(u_{i p}-u_{i l}\right)\right|^{\frac{2 N}{N+2}}\right)^{\frac{N+2}{2 N}}$.

Moreover:

$$
\left\|m_{i}\left(u_{i p}-u_{i l}\right)\right\|_{L^{\frac{2 N}{N+2}\left(\mathbb{R}^{N}-B_{R}\right)}} \leq\left\|m_{i}\right\|_{L^{N / 2}\left(\mathbb{R}^{N}-B_{R}\right)}\left\|u_{i p}-u_{i l}\right\|_{L^{2^{*}}\left(\mathbb{R}^{N}-B_{R}\right)} .
$$

Since $\left(u_{i p}\right)_{p}$ is a bounded sequence in $V_{q_{i}}\left(\mathbb{R}^{N}\right)$ and so in $L^{2^{*}}\left(\mathbb{R}^{N}\right)$, we deduce that $\left\|u_{i p}-u_{i l}\right\|_{L^{2^{*}\left(\mathbb{R}^{N}-B_{R}\right)}}$ is uniformly bounded respect to $R$. Furthermore $m_{i} \in L^{N / 2}\left(\mathbb{R}^{N}\right)$ and we can choose $R$ sufficiently large to make $\left\|m_{i}\right\|_{L^{N / 2}\left(\mathbb{R}^{N}-B_{R}\right)}$ as small as we need. Thus there exists $R_{0}$ such that

$$
\left\|m_{i}\left(u_{i p}-u_{i l}\right)\right\|_{L^{\frac{2 N}{N+2}}\left(\mathbb{R}^{N}-B_{R}\right)} \leq \epsilon \text { for all } n, p \text { and } R \geq R_{0} .
$$

Moreover, from the convergence of the sequence $\left(u_{i p}\right)_{p}$ in $L^{\frac{2 N}{N+2}}\left(B_{R_{0}}\right)$, we get: $\left\|m_{i}\left(u_{i p}-u_{i l}\right)\right\|_{L^{\frac{2 N}{N+2}}\left(B_{R_{0}}\right)} \leq\left\|m_{i}\right\|_{L^{\infty}\left(B_{R_{0}}\right)}\left\|u_{i p}-u_{i l}\right\|_{L^{\frac{2 N}{N+2}}\left(B_{R_{0}}\right)} \leq \epsilon$ for $p, l$ sufficiently large.

If the coefficient $m_{i j} \in L^{\infty}\left(\mathbb{R}^{N}\right)$ (similarly the weight $m_{i}$ ) then:

$\int_{\mathbb{R}^{N}} m_{i j}\left(u_{j p}-u_{j l}\right)\left(T_{i} u_{p}-T_{i} u_{l}\right) \leq c s t\left\|m_{i j}\right\|_{L^{\infty}\left(\mathbb{R}^{N}\right)}\left\|u_{j p}-u_{j l}\right\|_{L^{2}\left(\mathbb{R}^{N}\right)}\left\|T_{i} u_{p}-T_{i} u_{l}\right\|_{q_{i}}$.

Since the embedding of $V_{q_{j}}\left(\mathbb{R}^{N}\right)$ into $L^{2}\left(\mathbb{R}^{N}\right)$ is compact, there exists a subsequence of $\left(u_{j p}\right)_{p}$, still denoted by $\left(u_{j p}\right)_{p}$, which is a convergent sequence in $L^{2}\left(\mathbb{R}^{N}\right)$. Therefore we obtain the compactness of the operator $T$. 
We can conclude for the rest of the proof as in [7, Theorem 2.5]. Since the matrix $M$ is assumed to be symmetric, the operator $T$ is selfadjoint. So the largest eigenvalue of $T$ is given by:

$$
\mu_{1, M}=\sup _{u \in V, u \neq 0} \frac{\left\langle T u, u>_{V}\right.}{<u, u>_{V}}=\sup _{u \in V, u \neq 0} \frac{\sum_{i=1}^{n} \int_{\mathbb{R}^{N}} m_{i} u_{i}^{2}+\sum_{i, j=1 ; i \neq j}^{n} \int_{\mathbb{R}^{N}} m_{i j} u_{j} u_{i}}{\sum_{i=1}^{n} \int_{\mathbb{R}^{N}}\left[\left|\nabla u_{i}\right|^{2}+q_{i} u_{i}^{2}\right]} .
$$

Choosing $u=\left(u_{1}, \cdots, u_{n}\right) \in V$ such that supp $u_{i} \subset\left\{x \in \mathbb{R}^{N}, m_{i}(x)>0\right\}$ for one $i$ and $u_{j}=0$ if $j \neq i$, we get that $\mu_{1, M}>0$.

Now, we prove that $\Lambda_{1, M}=\frac{1}{\mu_{1, M}}$. We have $L^{-1} M \Phi_{1, M}=r\left(L^{-1} M\right) \Phi_{1, M}$ or equivalently $L \Phi_{1, M}=\Lambda_{1, M} M \Phi_{1, M}$. Therefore for all $i=1, \cdots, n$ :

$$
\left(-\Delta+q_{i}\right) \phi_{i, M}=\Lambda_{1, M}\left(m_{i} \phi_{i, M}+\sum_{j=1 ; j \neq i} m_{i j} \phi_{j, M}\right) \text { in } \mathbb{R}^{N} .
$$

Thus for all $v=\left(v_{1}, \cdots, v_{n}\right) \in V$, we have:

$$
\sum_{i=1}^{n} \int_{\mathbb{R}^{N}}\left[\nabla \phi_{i, M} . \nabla v_{i}+q_{i} \phi_{i, M} v_{i}\right]=\Lambda_{1, M} \sum_{i=1}^{n}\left(\int_{\mathbb{R}^{N}} m_{i} \phi_{i, M} v_{i}+\sum_{j=1 ; j \neq i}^{n} \int_{\mathbb{R}^{n}} m_{i j} \phi_{j, M} v_{i}\right) .
$$

For $v_{i}=\phi_{i, M}$, we get:

$$
\frac{1}{\Lambda_{1, M}}=\frac{\sum_{i=1}^{n} \int_{\mathbb{R}^{N}} m_{i} \phi_{i, M}^{2}+\sum_{i, j=1 ; i \neq j}^{n} \int_{\mathbb{R}^{N}} m_{i j} \phi_{j, M} \phi_{i, M}}{\sum_{i=1}^{n} \int_{\mathbb{R}^{N}}\left[\left|\nabla \phi_{i, M}\right|^{2}+q_{i} \phi_{i, M}^{2}\right]} \leq \mu_{1, M} .
$$

Moreover, since $\mu_{1, M}$ is an eigenvalue of the operator $T$ defined above, let $\psi=$ $\left(\psi_{1}, \cdots, \psi_{n}\right)$ be an eigenfunction associated with $\mu_{1, M}$. Since $T \psi=\mu_{1, M} \psi$, we have for all $v \in V$ :

$\mu_{1, M}<\psi, v>_{V}=<T \psi, v>_{V}=\beta(\psi, v)$ and so

$$
\mu_{1, M} \sum_{i=1}^{n} \int_{\mathbb{R}^{N}}\left[\nabla \psi_{i} . \nabla v_{i}+q_{i} \psi_{i} v_{i}\right]=\sum_{i=1}^{n} \int_{\mathbb{R}^{N}} m_{i} \psi_{i} v_{i}+\sum_{i, j=1 ; j \neq i}^{n} \int_{\mathbb{R}^{n}} m_{i j} \psi_{j} v_{i} .
$$

For $v=\left(0, \cdots, 0, v_{i}, 0, \cdots, 0\right) \in V$, we get:

$$
\int_{\mathbb{R}^{N}}\left[\nabla \psi_{i} . \nabla v_{i}+q_{i} \psi_{i} v_{i}\right]=\frac{1}{\mu_{1, M}}\left(\int_{\mathbb{R}^{N}} m_{i} \psi_{i} v_{i}+\sum_{j=1 ; j \neq i}^{n} \int_{\mathbb{R}^{n}} m_{i j} \psi_{j} v_{i}\right) .
$$

Therefore we have $L \psi=\frac{1}{\mu_{1, M}} M \psi$ or equivalently $L^{-1} M \psi=\mu_{1, M} \psi$. Thus $\mu_{1, M}$ is an eigenvalue of the operator $L^{-1} M$ and

$$
0<\mu_{1, M} \leq r\left(L^{-1} M\right)=\frac{1}{\Lambda_{1, M}} .
$$

From (3.1) and (3.2), we deduce that $\mu_{1, M}=\frac{1}{\Lambda_{1, M}}$ and $\Lambda_{1, M}$ satisfies (2.3).

Now, for indefinite and non necessarily bounded weights $m_{i}$, we prove the existence and uniqueness of a principal positive eigenvalue for (1.1) which extends the Theorem 2.6 in [7] to the case of non necessarily bounded weights and coefficients of the matrix $M$. Indeed, since $N \geq 3$, we have $H^{1}\left(\mathbb{R}^{N}\right) \subset L^{2^{*}}\left(\mathbb{R}^{N}\right)$ with a continuous embedding and so $V_{q_{i}}\left(\mathbb{R}^{N}\right) \subset L^{2^{*}}\left(\mathbb{R}^{N}\right)$ with a continuous embedding too and this allows us to work with weights $m_{i} \in L^{N / 2}\left(\mathbb{R}^{N}\right)$ and coefficients $m_{i j} \in L^{N / 2}\left(\mathbb{R}^{N}\right)$ 
contrary to [7] where we had $m_{i} \in L^{\infty}\left(\mathbb{R}^{N}\right)$ and $m_{i j} \in L^{\infty}\left(\mathbb{R}^{N}\right)$. This is the following result.

Theorem 3.2. Assume that each of the potentials $q_{i}$ satisfy $\left(\mathbf{H}_{\mathbf{q}}^{\mathbf{1}}\right)$ and $\left(\mathbf{H}_{\mathbf{q}}^{\mathbf{2}}\right)$ and each of the weights $m_{i}$ satisfy $\left(\mathbf{H}_{\mathbf{m}}^{\mathbf{3}}\right)$. Assume also that $(\mathbf{H 4})-(\mathbf{H 6})$ are satisfied. Then there exists a unique principal eigenvalue $\Lambda_{1, M}>0$ associated with a positive eigenfunction $\Phi_{1, M}=\left(\phi_{1, M}, \cdots, \phi_{n, M}\right) \in V:=V_{q_{1}}\left(\mathbb{R}^{N}\right) \times \cdots \times V_{q_{n}}\left(\mathbb{R}^{N}\right), \phi_{i, M}>0$ and $\Lambda_{1, M}$ satisfies $(2.3)$.

Proof. We follow a method developed in [15] (for an equation in a bounded domain) and in [7, Theorem 2.6]. Let $\Omega_{i}^{+}=\left\{x \in \mathbb{R}^{N}, m_{i}(x)>0\right\}$, meas $\left(\Omega_{i}^{+}\right)>0$, $\Omega_{i}^{-}=\left\{x \in \mathbb{R}^{N}, m_{i}(x)<0\right\}$, meas $\left(\Omega_{i}^{-}\right)>0$, and $\Omega_{i}^{0}=\left\{x \in \mathbb{R}^{N}, m_{i}(x)=0\right\}$. Let $U=\left(u_{1}, \cdots, u_{n}\right)$ be a solution of (1.1). Let $h>0, h$ a fixed function which satisfies $h \in L^{N}\left(\mathbb{R}^{N}\right) \cap L^{N / 2}\left(\mathbb{R}^{N}\right) \cap L^{\infty}\left(\mathbb{R}^{N}\right)$. We have for all $i$ :

$$
\left(-\Delta+q_{i}\right) u_{i}+\lambda m_{i}^{-} u_{i}=\lambda\left(m_{i}^{+} u_{i}+\sum_{j=1 ; j \neq i}^{n} m_{i j} u_{j}\right) \text { in } \mathbb{R}^{N} .
$$

For given $\lambda \geq 0$, we rewrite (3.3) as an eigenvalue problem with parameter $\sigma(\lambda)$. For all $i$,

$$
\left(-\Delta+q_{i}\right) u_{i}+\lambda\left(m_{i}^{-}+1_{i} h\right) u_{i}=\sigma(\lambda)\left(\left(m_{i}^{+}+1_{i} h\right) u_{i}+\sum_{j=1 ; j \neq i}^{n} m_{i j} u_{j}\right) \text { in } \mathbb{R}^{N}
$$

where $1_{i}$ denotes the characteristic function of $\Omega_{i}^{0} \cup \Omega_{i}^{-}$. We denote by $Q_{i, \lambda}:=$ $q_{i}+\lambda\left(m_{i}^{-}+1_{i} h\right)$ and $\rho_{i}:=m_{i}^{+}+1_{i} h$. Then (3.4) is equivalent to

$$
\left(-\Delta+Q_{i, \lambda}\right) u_{i}=\sigma(\lambda)\left(\rho_{i} u_{i}+\sum_{j=1 ; j \neq i}^{n} m_{i j} u_{j}\right) \text { in } \mathbb{R}^{N}
$$

Note that the weight $\rho_{i}>0$ in $\mathbb{R}^{N}, \rho_{i}$ satisfies $\left(\mathbf{H}_{\mathbf{m}}^{\mathbf{3}}\right)$ and the potential $Q_{i, \lambda}$ satisfies $\left(\mathbf{H}_{\mathbf{q}}^{\mathbf{1}}\right)$ and $\left(\mathbf{H}_{\mathbf{q}}^{\mathbf{2}}\right)$ since $\lambda \geq 0$. From Theorem 3.1, we deduce that the system (3.5) has a unique principal eigenvalue $\sigma(\lambda)$ associated with a principal eigenfunction $\Phi_{\lambda}=\left(\phi_{1, \lambda}, \cdots, \phi_{n, \lambda}\right), \phi_{i, \lambda}>0$. From $(2.3)$, we get:

$$
\begin{aligned}
\sigma(\lambda)= & \inf \left\{\frac{\sum_{i=1}^{n} \int_{\mathbb{R}^{N}}\left[\left|\nabla \phi_{i}\right|^{2}+q_{i} \phi_{i}^{2}\right]+\lambda \sum_{i=1}^{n} \int_{\mathbb{R}^{N}}\left(m_{i}^{-}+1_{i} h\right) \phi_{i}^{2}}{\sum_{i=1}^{n} \int_{\mathbb{R}^{N}}\left(m_{i}^{+}+1_{i} h\right) \phi_{i}^{2}+\sum_{i, j=1 ; i \neq j}^{n} \int_{\mathbb{R}^{N}} m_{i j} \phi_{i} \phi_{j}}, \phi=\left(\phi_{1}, \cdots, \phi_{n}\right) \in V\right. \\
& \text { such that } \left.\sum_{i=1}^{n} \int_{\mathbb{R}^{N}}\left(m_{i}^{+}+1_{i} h\right) \phi_{i}^{2}+\sum_{i, j=1 ; i \neq j}^{n} \int_{\mathbb{R}^{N}} m_{i j} \phi_{i} \phi_{j}>0\right\} .
\end{aligned}
$$

Therefore, $\sigma(\lambda)<\Lambda_{1, Q, N}^{+}$the principal eigenvalue of the operator $L_{Q}$ associated with the matrix $N=\left(n_{i j}\right)$ where $L_{Q}=\operatorname{diag}\left(-\Delta+Q_{i, \lambda}\right), n_{i i}=\rho_{i}$ and $n_{i j}=m_{i j}$ in $\Omega=\cap_{i=1}^{n} \Omega_{i}^{+}$with Dirichlet boundary condition. Note that $\Lambda_{1, Q, N}^{+}$is in fact independant of $\lambda$. Therefore the function $\sigma$ is bounded. Note that $\sigma: \lambda \mapsto \sigma(\lambda)$ is increasing and continuous and that $\sigma(0)>0$. Therefore for all $\lambda>0$, we have $0<\sigma(0)<\sigma(\lambda)<\Lambda_{1, Q, N}^{+}$. Thus we deduce that there exists $0<\tilde{\lambda}<\Lambda_{1, Q, N}^{+}$such that $\sigma(\tilde{\lambda})=\tilde{\lambda}$. Proceeding as in [15], we can show that $\tilde{\lambda}$ is unique.

Indeed, let $\lambda>\mu>0$. If we denote by $\Phi_{\lambda}=\left(\phi_{1, \lambda}, \cdots, \phi_{n, \lambda}\right), \phi_{i, \lambda}>0$, and by 
$\Phi_{\mu}=\left(\phi_{1, \mu}, \cdots, \phi_{n, \mu}\right), \phi_{i, \mu}>0$ the associated eigenfunctions with $\sigma(\lambda)$ and $\sigma(\mu)$, from the variational characterization (3.6), since $\lambda \neq \mu$ we have:

$$
\frac{\sigma(\lambda)-\sigma(\mu)}{\lambda-\mu} \leq \frac{\sum_{i=1}^{n} \int_{\mathbb{R}^{N}}\left(m_{i}^{-}+1_{i} h\right) \phi_{i, \mu}^{2}}{\sum_{i=1}^{n} \int_{\mathbb{R}^{N}}\left(m_{i}^{+}+1_{i} h\right) \phi_{i, \mu}^{2}+\sum_{i, j=1 ; i \neq j}^{n} \int_{\mathbb{R}^{N}} m_{i j} \phi_{i, \mu} \phi_{j, \mu}} .
$$

For $\mu=\tilde{\lambda}<\lambda$, we get

$$
\frac{\sigma(\lambda)-\tilde{\lambda}}{\lambda-\tilde{\lambda}} \leq \frac{\sum_{i=1}^{n} \int_{\mathbb{R}^{N}}\left(m_{i}^{-}+1_{i} h\right) \phi_{i, \tilde{\lambda}}^{2}}{\sum_{i=1}^{n} \int_{\mathbb{R}^{N}}\left(m_{i}^{+}+1_{i} h\right) \phi_{i, \tilde{\lambda}}^{2}+\sum_{i, j=1 ; i \neq j}^{n} \int_{\mathbb{R}^{N}} m_{i j} \phi_{i, \tilde{\lambda}} \phi_{j, \tilde{\lambda}}}<1 .
$$

And so $\sigma(\lambda)<\lambda$ for $\lambda>\tilde{\lambda}$. In the same way we show that $\sigma(\lambda)>\lambda$ for $\lambda<\tilde{\lambda}$ and this gives the uniqueness of $\tilde{\lambda}$.

Now, we verify that $\tilde{\lambda}$ satisfies (2.3). We proceed exactly as in [7, Theorem 2.6]. Let us denote by

$$
\begin{aligned}
\lambda^{*}=\inf \left\{\frac{\sum_{i=1}^{n} \int_{\mathbb{R}^{N}}\left[\left|\nabla \phi_{i}\right|^{2}+q_{i} \phi_{i}^{2}\right]}{\sum_{i=1}^{n} \int_{\mathbb{R}^{N}} m_{i} \phi_{i}^{2}+\sum_{i, j=1 ; i \neq j}^{n} \int_{\mathbb{R}^{N}} m_{i j} \phi_{i} \phi_{j}}, \phi=\left(\phi_{1}, \cdots, \phi_{n}\right) \in V\right. \\
\left.\quad \text { such that } \sum_{i=1}^{n} \int_{\mathbb{R}^{N}} m_{i} \phi_{i}^{2}+\sum_{i, j=1 ; i \neq j}^{n} \int_{\mathbb{R}^{N}} m_{i j} \phi_{i} \phi_{j}>0\right\} .
\end{aligned}
$$

Since

$$
\tilde{\lambda}=\frac{\sum_{i=1}^{n} \int_{\mathbb{R}^{N}}\left[\left|\nabla \phi_{i, \tilde{\lambda}}\right|^{2}+q_{i} \phi_{i, \tilde{\lambda}}^{2}\right]+\tilde{\lambda} \sum_{i=1}^{n} \int_{\mathbb{R}^{N}}\left(m_{i}^{-}+1_{i} h\right) \phi_{i, \tilde{\lambda}}^{2}}{\sum_{i=1}^{n} \int_{\mathbb{R}^{N}}\left(m_{i}^{+}+1_{i} h\right) \phi_{i, \tilde{\lambda}}^{2}+\sum_{i, j=1 ; i \neq j}^{n} \int_{\mathbb{R}^{N}} m_{i j} \phi_{i, \tilde{\lambda}} \phi_{j, \tilde{\lambda}}}
$$

we have $\tilde{\lambda} \geq \lambda^{*}$.

Moreover let $\phi=\left(\phi_{1}, \cdots, \phi_{n}\right) \in V$ be such that

$$
\sum_{i=1}^{n} \int_{\mathbb{R}^{N}} m_{i} \phi_{i}^{2}+\sum_{i, j=1 ; i \neq j}^{n} \int_{\mathbb{R}^{N}} m_{i j} \phi_{i} \phi_{j}>0
$$

Since $\tilde{\lambda}=\sigma(\tilde{\lambda})$, we get

$$
\tilde{\lambda} \leq \frac{\sum_{i=1}^{n} \int_{\mathbb{R}^{N}}\left[\left|\nabla \phi_{i}\right|^{2}+q_{i} \phi_{i}^{2}\right]}{\sum_{i=1}^{n} \int_{\mathbb{R}^{N}} m_{i} \phi_{i}^{2}+\sum_{i, j=1 ; i \neq j}^{n} \int_{\mathbb{R}^{N}} m_{i j} \phi_{i} \phi_{j}} .
$$

Thus $\tilde{\lambda} \leq \lambda^{*}$.

As in [7], note that for all $i=1, \cdots, n, \Lambda_{1, M}<\lambda_{1, q_{i}, m_{i}}$.

Indeed, from (2.1) and (2.3), we have $\Lambda_{1, M} \leq \lambda_{1, q_{i}, m_{i}}$. Suppose that $\Lambda_{1, M}=$ $\lambda_{1, q_{i}, m_{i}}$. Then we have:

$$
\left(-\Delta+q_{i}\right)\left(\phi_{i, M}-\phi_{1, q_{i}, m_{i}}\right)=\lambda_{1, q_{i}, m_{i}} m_{i}\left(\phi_{i, M}-\phi_{1, q_{i}, m_{i}}\right)+\lambda_{1, q_{i}, m_{i}} \sum_{j=1 ; j \neq i}^{n} m_{i j} \phi_{j, M} \text { in } \mathbb{R}^{N} \text {. }
$$

Multiplying by $\phi_{1, q_{i}, m_{i}}$ and integrating over $\mathbb{R}^{N}$, we obtain (since $\lambda_{1, q_{i}, m_{i}}>0$ ), $\int_{\mathbb{R}^{N}} \sum_{j=1 ; j \neq i}^{n} m_{i j} \phi_{j, M} \phi_{1, q_{i}, m_{i}}=0$. Since $m_{i j}>0, \phi_{i, M}>0$ and $\phi_{1, q_{i}, m_{i}}>0$ we get a contradiction. 


\section{SOME TWO-BY-TWO NON COOPERATIVE SYSTEMS}

We will study in this section the existence of a global positive eigenvalue $\Lambda_{1, M}$ associated with a positive eigenfunction $\Phi_{1, M}$ (i.e. a pincipal positive eigenvalue) in the case of two $2 \times 2$ non cooperative systems. In a first example, we study a non cooperative system with constant coefficients and with the same potential $q:=q_{1}=q_{2}$. So we rewrite (1.1) under the following form

$$
\left\{\begin{aligned}
(-\Delta+q) u & =\lambda(a u+b v) \text { in } \mathbb{R}^{N} \\
(-\Delta+q) v & =\lambda(c u+d v) \text { in } \mathbb{R}^{N}
\end{aligned}\right.
$$

where the constant coefficients satisfy the following hypotheses

$$
\text { (H7): }\left\{\begin{array}{l}
a \in \mathbb{R}, a>2 ; b \in \mathbb{R}, b<0,|b|<\sqrt{a} . \\
c=b+k+\frac{b+k}{n} \text { with } k=\frac{-b+\sqrt{b^{2}+4(a-2)}}{2}>0 \text { and } n \in \mathbb{R}, n>3 a-1 . \\
d=\frac{1}{k}\left(b+k-\frac{b+k}{n}\left(1+k^{2}\right)\right) .
\end{array}\right.
$$

The idea here is to insert this non cooperative $2 \times 2$ system $(4.1)(b<0)$ into a strictly cooperative and symmetric $3 \times 3$ one. But we cannot follow the insertion used in $[13,8]$ because we need to obtain a $3 \times 3$ symmetric system with strictly positive off-diagonal coefficients in order to apply Theorems 2.3 or 2.4 . We have the following result

Theorem 4.1. Assume that the potential $q$ satisfies $\left(\mathbf{H}_{\mathbf{q}}^{\mathbf{1}}\right)$ and $\left(\mathbf{H}_{\mathbf{q}}^{\mathbf{2}}\right)$. Assume also that (H7) is satisfied. Then there exists a principal positive eigenvalue $\Lambda_{1, M}>0$ associated with a positive eigenfunction $\Phi_{1, M}=\left(\phi_{1, M}, \phi_{2, M}\right) \in V:=V_{q}\left(\mathbb{R}^{N}\right) \times$ $V_{q}\left(\mathbb{R}^{N}\right), \phi_{i, M}>0$ for the system (4.1).

Proof. First note that $b+k>0$ (and so $c>0$ ). Also note that $d>0$ (since $n>3 a-1)$. Let $(u, v)$ be a solution of $(4.1)$ and let $w=u-k v$. Then $(u, v, w)$ satisfies the following system

$$
\left\{\begin{array}{l}
(-\Delta+q) u=\lambda((a-1) u+(b+k) v+w) \text { in } \mathbb{R}^{N} \\
(-\Delta+q) v=\lambda\left((b+k) u+\left(d+k \frac{b+k}{n}\right) v+\frac{b+k}{n} w\right) \text { in } \mathbb{R}^{N} \\
(-\Delta+q) w=\lambda\left(u+\frac{b+k}{n} v+\left(1-k \frac{b+k}{n}\right) w\right) \text { in } \mathbb{R}^{N} .
\end{array}\right.
$$

Moreover $1-k \frac{b+k}{n}>0$. Since the matrix of the system (4.2) is symmetric and with strictly positive coefficients, from the Theorem 2.3 we get the existence of a positive principal eigenvalue $\Lambda_{1, M}>0$ associated with a positive eigenfunction $\Phi_{1, M}=\left(\phi_{1, M}, \phi_{2, M}, \phi_{2, M}\right) \in V:=V_{q}\left(\mathbb{R}^{N}\right) \times V_{q}\left(\mathbb{R}^{N}\right) \times V_{q}\left(\mathbb{R}^{N}\right), \phi_{i, M}>0$ for the system (4.2). And we have

$$
\left\{\begin{array}{l}
(-\Delta+q) \phi_{1, M}=\Lambda_{1, M}\left((a-1) \phi_{1, M}+(b+k) \phi_{2, M}+\phi_{3, M}\right) \text { in } \mathbb{R}^{N} \\
(-\Delta+q) \phi_{2, M}=\Lambda_{1, M}\left((b+k) \phi_{1, M}+\left(d+k \frac{b+k}{n}\right) \phi_{2, M}+\frac{b+k}{n} \phi_{3, M}\right) \text { in } \mathbb{R}^{N} \\
(-\Delta+q) \phi_{3, M}=\Lambda_{1, M}\left(\phi_{1, M}+\frac{b+k}{n} \phi_{2, M}+\left(1-k \frac{b+k}{n}\right) \phi_{3, M}\right) \text { in } \mathbb{R}^{N} .
\end{array}\right.
$$

Denote by $\phi=\phi_{1, M}-k \phi_{2, M}$. Then

$(-\Delta+q) \phi=\Lambda_{1, M}\left((a-1-k(b+k)) \phi_{1, M}+\left(b+k-k\left(d+k \frac{b+k}{n}\right)\right) \phi_{2, M}+\left(1-k \frac{b+k}{n}\right) \phi_{3, M}\right)$ in $\mathbb{R}^{N}$.

Therefore

$(-\Delta+q) \phi=\Lambda_{1, M}\left(\phi_{1, M}+\frac{b+k}{n} \phi_{2, M}+\left(1-k \frac{b+k}{n}\right) \phi_{3, M}\right)=(-\Delta+q) \phi_{3, M}$ in $\mathbb{R}^{N}$. 
So $\phi=\phi_{3, M}$ and $\left(\phi_{1, M}, \phi_{2, M}\right)$ satisfies

$$
\left\{\begin{array}{l}
(-\Delta+q) \phi_{1, M}=\Lambda_{1, M}\left(a \phi_{1, M}+b \phi_{2, M}\right) \text { in } \mathbb{R}^{N} \\
(-\Delta+q) \phi_{2, M}=\Lambda_{1, M}\left(c \phi_{1, M}+d \phi_{2, M}\right) \text { in } \mathbb{R}^{N} .
\end{array}\right.
$$

We now consider as a last example of this section the following system:

$$
\left\{\begin{aligned}
\left(-\Delta+q_{1}\right) u & =\lambda(a u+b v) \text { in } \mathbb{R}^{N} \\
\left(-\Delta+q_{2}\right) v & =\lambda(c u+d v) \text { in } \mathbb{R}^{N}
\end{aligned}\right.
$$

where the coefficients $a, b, c, d$ are bounded. The ideas, here, are to decouple the system in order to diagonalise the matrix $M$. We denote by $M(x)=\left(\begin{array}{cc}a(x) & b(x) \\ c(x) & d(x)\end{array}\right)$ the coupling matrix of the coefficients of the system (4.3). Following [10], we introduce $S$ an invertible $2 \times 2$ matrix of constants such that $S$ diagonalises $M(x)$ for all $x$. In [10], it is proved that such a choice is possible only if either (case I) $b(x)$ and $c(x)$ are both multiples of $a(x)-d(x)$ or (case II) $a(x)=d(x)$ for all $x$ and $b(x)$ and $c(x)$ are positive multiples of each other. We will only consider the case I in order to have a non cooperative system. We define the functions $u^{*}$ and $v^{*}$ by $\left(\begin{array}{c}u^{*} \\ v^{*}\end{array}\right)=S^{-1}\left(\begin{array}{c}u \\ v\end{array}\right)$ and since $S$ is a constant matrix, we obtain

$$
\left(\begin{array}{cc}
-\Delta+q_{1} & 0 \\
0 & -\Delta+q_{2}
\end{array}\right)\left(\begin{array}{l}
u^{*} \\
v^{*}
\end{array}\right)=\lambda S^{-1} M(x) S\left(\begin{array}{c}
u^{*} \\
v^{*}
\end{array}\right) .
$$

We suppose that the coefficients $a, b, c, d$ of the system satisfy the following hypotheses:

$$
\text { (H8): }\left\{\begin{array}{l}
(i) a, b, c, d \in L^{\infty}\left(\mathbb{R}^{N}\right) . \\
(i i) b \text { and } c \text { are positive multiples of } a-d .
\end{array}\right.
$$

We rewrite the matrix $M(x)$ under the following forms:

$$
M(x)=\left(\begin{array}{cc}
a(x) & b^{*}(a(x)-d(x)) \\
c^{*}(a(x)-d(x)) & d(x)
\end{array}\right)
$$

where $a \neq d$ and $b^{*}$ and $c^{*}$ are constants.

Following [10], we can decouple the system (4.4) if and only if $1+4 b^{*} c^{*} \geq 0$.

If $1+4 b^{*} c^{*}>0$ and $b^{*}<0, c^{*}>0$, we define the two positive constants $\rho_{1}=$ $\frac{1+\sqrt{1+4 b^{*} c^{*}}}{2}$ and $\rho_{2}=\frac{1-\sqrt{1+4 b^{*} c^{*}}}{2}$. We choose $S=\left(\begin{array}{cc}-b^{*} & -b^{*} \\ \rho_{1} & \rho_{2}\end{array}\right)$ and we have $u=-b^{*}\left(u^{*}+v^{*}\right)$ and $v=\rho_{1} u^{*}+\rho_{2} v^{*}$. Now, if we define the functions

$$
\begin{aligned}
& \mu_{1}(x):=\frac{1}{\rho_{1}-\rho_{2}}\left[\rho_{1} d(x)-\rho_{2} a(x)+2 \rho_{1} \rho_{2}(a(x)-d(x))\right] \\
& \mu_{2}(x):=\frac{1}{\rho_{1}-\rho_{2}}\left[\rho_{1} a(x)-\rho_{2} d(x)-2 \rho_{1} \rho_{2}(a(x)-d(x))\right],
\end{aligned}
$$

then we can write the decoupled system as

$$
\left\{\begin{array}{l}
\left(-\Delta+q_{1}\right) u^{*}=\lambda \mu_{1} u^{*} \text { in } \mathbb{R}^{N} \\
(-\Delta+q)_{2} v^{*}=\lambda \mu_{2} v^{*} \text { in } \mathbb{R}^{N}
\end{array}\right.
$$

Therefore we have the following result 
Theorem 4.2. Assume that each of the potential $q_{i}$ satisfy $\left(\mathbf{H}_{\mathbf{q}}^{\mathbf{1}}\right)$. Assume also that (H8) is satisfied. Then if $\lambda_{1, q_{1}, \mu_{1}}=\lambda_{1, q_{2}, \mu_{2}}$ (resp. if $\tilde{\lambda}_{1, q_{1}, \mu_{1}}=\tilde{\lambda}_{1, q_{2}, \mu_{2}}$ ) there exists a principal positive (resp. negative) eigenvalue $\Lambda_{1, M}$ associated with a positive eigenfunction $\Phi_{1, M}=\left(\phi_{1, M}, \phi_{2, M}\right) \in V:=V_{q_{1}}\left(\mathbb{R}^{N}\right) \times V_{q_{2}}\left(\mathbb{R}^{N}\right), \phi_{i, M}>0$ for the system (4.3), where $\lambda_{1, q_{i}, \mu_{i}}$ (resp. $\tilde{\lambda}_{1, q_{i}, \mu_{i}}$ ) is the unique positive (resp. negative) principal eigenvalue for the operator $-\Delta+q_{i}$ associated with the potential $\mu_{i}$ defined by (4.5) and (4.6).

Proof. Assume that for example $\lambda_{1, q_{1}, \mu_{1}}=\lambda_{1, q_{2}, \mu_{2}}$. Then from Theorems 2.1, 2.2 there exists $\phi_{1, q_{1}, \mu_{1}}>0$ a positive eigenfunction associated with the eigenvalue $\lambda_{1, q_{1}, \mu_{1}}$ and there exists $\phi_{1, q_{2}, \mu_{2}}>0$ a positive eigenfunction associated with the eigenvalue $\lambda_{1, q_{2}, \mu_{2}}=\lambda_{1, q_{1}, \mu_{1}}$. Then $\left(\phi_{1, q_{1}, \mu_{1}}, \phi_{1, q_{2}, \mu_{2}}\right)$ is a solution of (4.7). Let $\phi_{1, M}=-b^{*}\left(\phi_{1, q_{1}, \mu_{1}}+\phi_{1, q_{2}, \mu_{2}}\right)>0$ and $\phi_{2, M}=\rho_{1} \phi_{1, q_{1}, \mu_{1}}+\rho_{2} \phi_{1, q_{2}, \mu_{2}}>0$. We get that $\left(\phi_{1, M}, \phi_{2, M}\right)$ is a positive solution of (4.3) associated with the positive eigenvalue $\lambda_{1, q_{1}, \mu_{1}}$.

\section{REFERENCES}

[1] Alziary, B.-Fleckinger, J.-Lécureux, M.-H.: Systems of Schrödinger Equations. Positivity and Negativity, Monografiás del Seminario Matemático García de Galdeano 33 (2006), 19-26.

[2] Alziary, B.-Fleckinger, J.-Takác,P.: An Extension of Maximum and Antimaximum Principles to a Schrödinger Equation in $\mathbb{R}^{2}$, Journal of Differential Equations 156 (1991), 122-152.

[3] Alziary,B.-Takác,P.: A Pointwise Lower Bound for Positive Solutions of a Schrödinger Equation in $\mathbb{R}^{N}$, Journal of Differential Equations 133 (2) (1997), 280-295.

[4] Brown, K.J.-Cosner, C.-Fleckinger,J.: Principal Eigenvalues for Problems with Indefinite Weight Function on $\mathbb{R}^{N}$, Proc. AMS 108 (1990), 147-155.

[5] Cantrell, R.S.-Schmitt,S.: On the Eigenvalue Problem for Coupled Elliptic Systems, SIAM J. Math. Anal. Vol.17 No.4 (1986), 850-862.

[6] Cardoulis, L: Schrödinger Equations with Indefinite Weights in the Whole Space, C. R. Acad. Sci. Paris, Ser I 347 (2009), 1255-1260.

[7] Cardoulis, L.: Systems of Schrödinger Equations in the Whole Space, Rostock. Math. Kolloq. 65 (2009), 29-49.

[8] Cardoulis, L.: Insertion of a Non Cooperative Elliptic System Involving Schrödinger Operators into a Cooperrative One, Mono. del Seminario "Garcia de Galdeano" 27 (2003), $169-176$.

[9] Clément, P.-Peletier, L.: An Antimaximum Principle for Second Order Elliptic Operators, J. Diff. Eq. 34 (1979), 218-229.

[10] Cosner, C.-Schaefer, P.W.: Sign-definite Solutions in Some Linear Elliptic Systems, Proc. Roy. Soc. Edinburgh 111A (1989), 347-358.

[11] Daners, D.-Koch-Medina, P.: Abstract Evolution Equations, Periodic Problems and Applications, Longman Research Notes 279 (1992).

[12] de Figueiredo, D.G.-Mitidieri, E.: A Maximum Principle for an Elliptic System and Applications to Semilinear Problems, S.I.A.M. J. Math. Anal. 17 (1986), 836-849.

[13] de Figueiredo, D.G.-Mitidieri, E.: Maximum Principle for Linear Elliptic Systems, Quaterno Matematico 177, Dip. Sc. Mat., Univ. Trieste, (1988).

[14] Fleckinger, J.-Hernández, J.- de Thélin, F.: On Maximum Principles and Existence of Positive Solutions for Some Cooperative Elliptic Systems, Diff. and Int. Eq. 8, N.1 (1995), $69-85$.

[15] Fleckinger, J.-Hernández, J.—de Thélin, F.: Existence of Multiple Principal Eigenvalues for some Indefinite Linear Eigenvalue Problems, Bollettino U.M.I. 8, 7B (2004), 159-188.

[16] Hess, P.: An Antimaximum Principle for Linear Elliptic Equations with an Indefinite Weight Function, J. Differential Equations 41 (1981), 369-374.

[17] Hess, P.: On the Eigenvalue Problem for Weakly Coupled Elliptic Systems, Arch. Rat. Mech. Anal. 81 (1985), 151-159.

[18] Sweers, G.: Strong Positivity in C $(\bar{\Omega})$ for Elliptic Systems, Math. Z. 209 (1992), 251-271. 
[19] Takác, P.: An Abstract Form of Maximum and Antimaximum Principles of Hopf's Type, J. Math. Appl. 201 (1996), 339-364.

* Université de Toulouse, institut Mathématiques de Toulouse, Ceremath/UmR MiP, 2 rue du Doyen Gabriel Marty, 31042 Toulouse cedex 9, France

E-mail address: laure.cardoulis@univ-tlse1.fr 